\title{
Gastric band cutter to remove a migrated gastric band
}

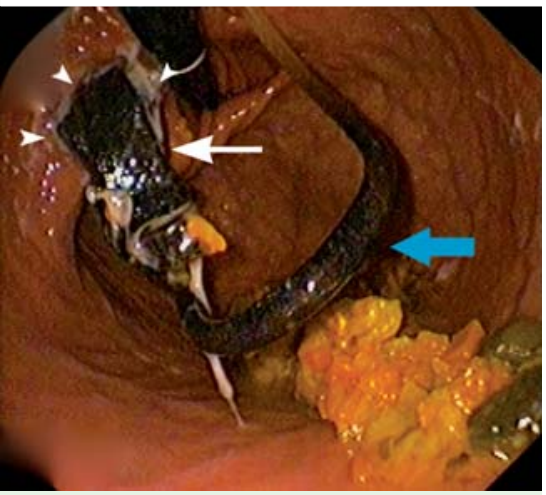

Fig. 1 Endoscopic image of the migrated gastric band in the subcardial gastric area. The band (white arrow) is in a small eroded tunnel in the gastric wall (arrow heads), while the connection tube (blue arrow) has partially migrated inside the gastric cavity.

Laparoscopic adjustable gastric banding is a popular method in bariatric surgery. A silicone ring is adjusted in the gastric fundus to create a smaller proximal compartment [1]. Intragastric migration of a gastric band is a common complication that might require surgical treatment.

A 36 year-old woman who underwent adjustable gastric band placement 7 years before was referred to our center due to intragastric band migration ( Fig.1). A specifically designed device (A.M.I. Gastric Band Cutter; CJ Medical, Haddenham, UK) ( Fig.2) was used for an endoscopic management approach. With the patient under general anesthesia, an upper endoscope was used to introduce the metallic thread between the gastric band and trapped gastric mucosa. The thread was then recaptured with a polypectomy snare in a parallel fashion, trapping the band. Both ends of the thread were pulled and attached to the tourniquet on the handgrip of the device. The external metal sheath was compressed on the gastric band while twisting the tourniquet, cutting the band by strangulation ( Fig.3). Surgical removal of the subcutaneous port and cutting of the connection tube were performed, releasing the band inside the stomach. The band was pulled out of the patient with the endoscope ( $\bullet$ Fig.4). The patient was discharged the next day without complications.
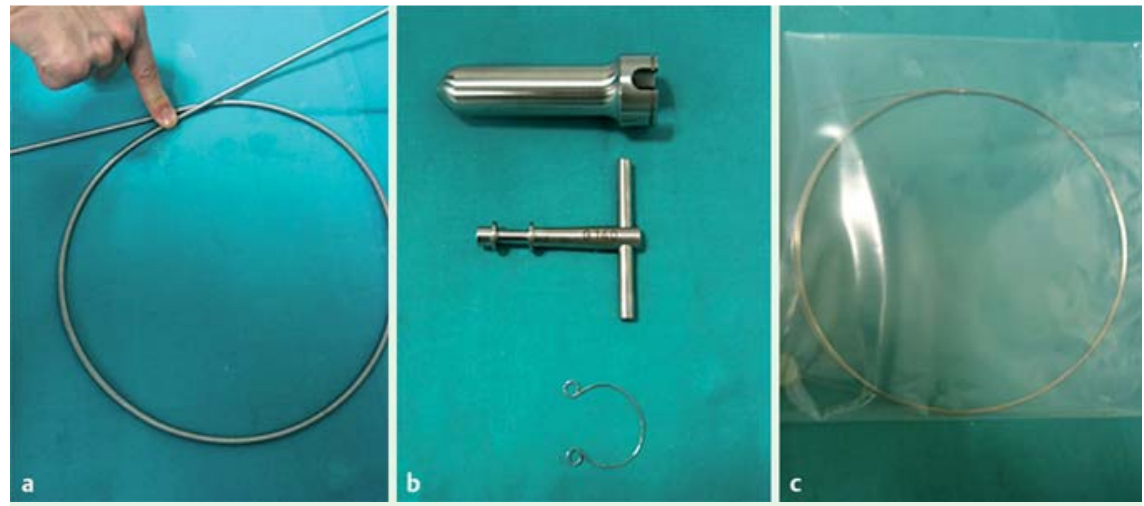

Fig. 2 Components of the A.M.I. Gastric Band Cutter: a metallic sheath; b handgrip with tourniquet; c metallic cutting thread.
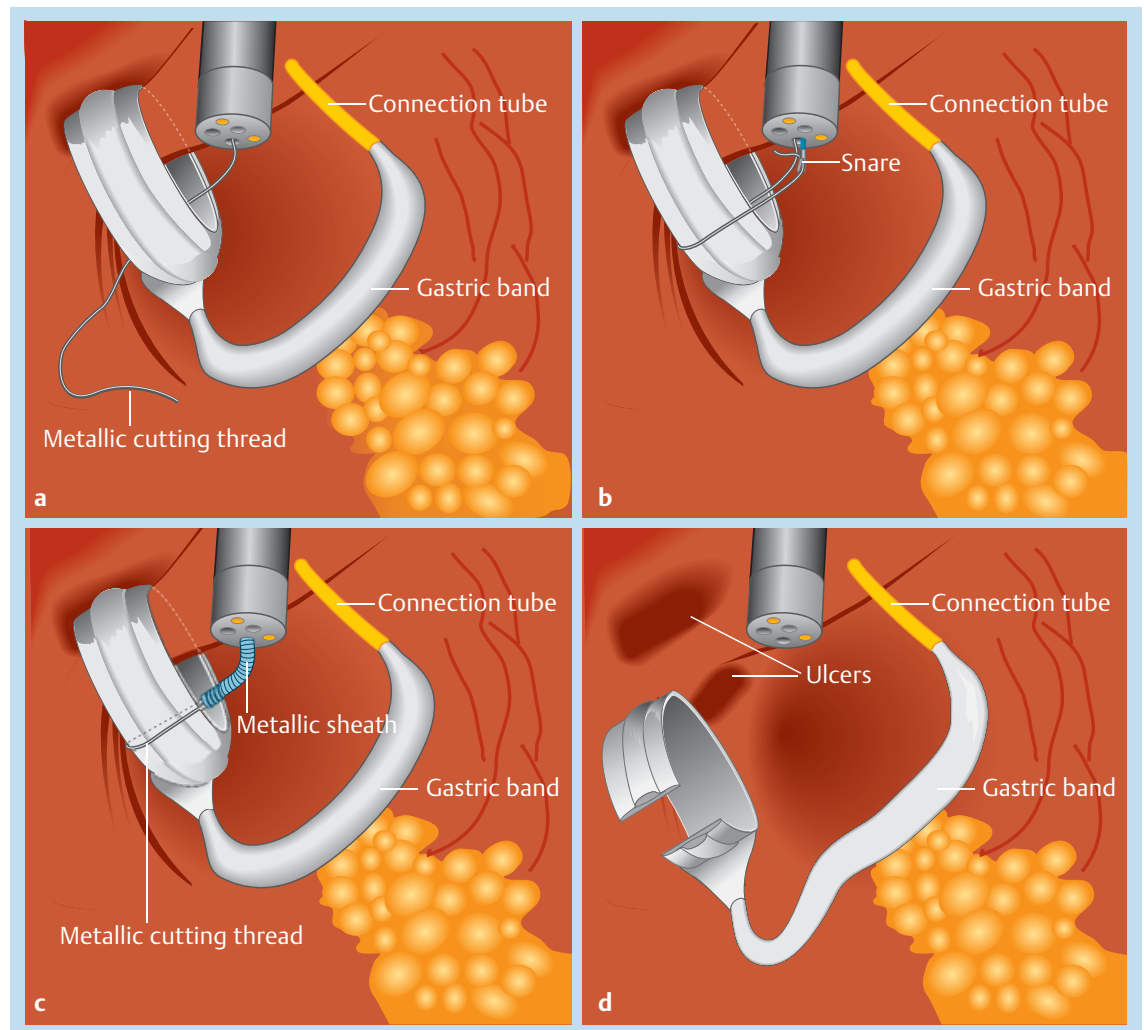

Fig. 3 Drawings showing the consecutive steps in the band-cutting process in the gastric fundus. a The metallic thread is introduced between the gastric band and trapped gastric mucosa. $\mathbf{b}$ The tip of the metallic thread is recaptured with a polypectomy snare in parallel fashion, trapping the gastric band with the thread. $\mathbf{c}$ Both ends of the thread are pulled and attached to the tourniquet on handgrip of the device, while the external metal sheath is advanced and compressed onto the gastric band. d Twisting the tourniquet cuts the band by strangulation. Eroded ulcers are visible where the gastric band was placed.

After a 12-month follow-up there had not been any relapse of the patient's symptoms or any complications.
Intragastric migration is a relatively common complication of gastric banding (in $0.5 \%-7 \%$ of cases) [2]. Endoscopic remov- 


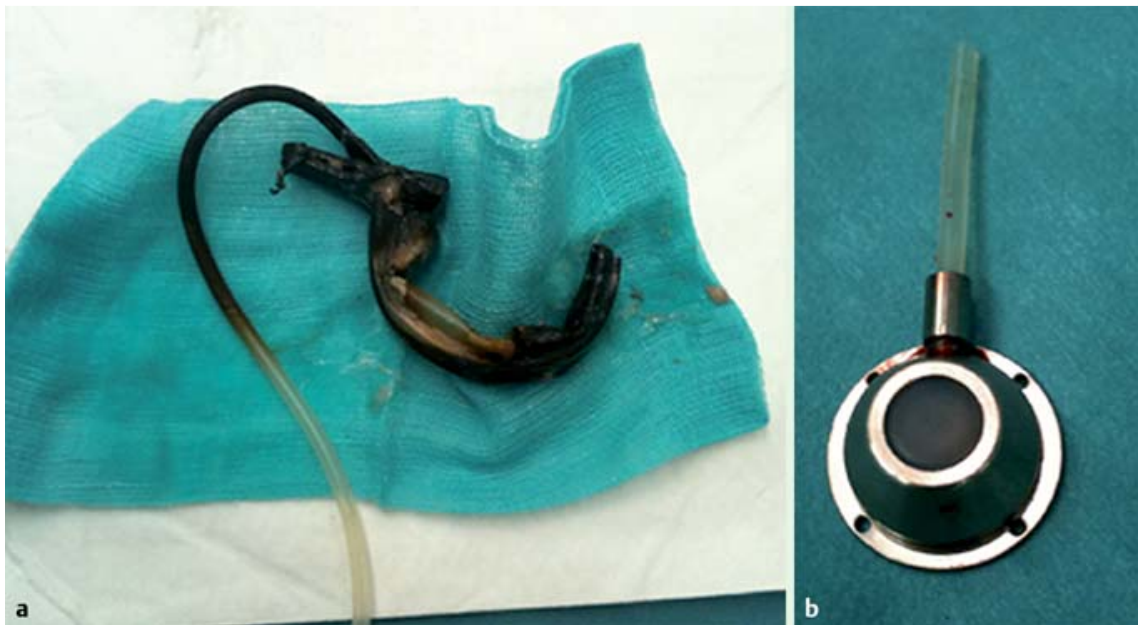

Fig. 4 a Cut gastric band. $\mathbf{b}$ Subcutaneous port exposed after removal from the patient.

al of a migrated gastric band has been described in the literature since $2000[3,4]$. This method seems, from this case, to be feasible and effective for initial minimally invasive management of gastric band migration.

Endoscopy_UCTN_Code_TTT_1AO_2AD

Competing interests: None
A. Herreros de Tejada ${ }^{1}$, J. L. Calleja ${ }^{1}$, M. Jiménez ${ }^{2}$, V. Rojo ${ }^{2}$, C. Santander ${ }^{3}$, J. C. Rial ${ }^{4}$, R. García ${ }^{1}$, J. Chennat ${ }^{5}$, A. L. Picardo ${ }^{6}$, L. Abreu ${ }^{1}$

${ }^{1}$ Department of Gastroenterology, Puerta de Hierro University Hospital, Majadahonda, Spain

${ }^{2}$ Department of Surgery, Puerta de Hierro University Hospital, Majadahonda, Spain

${ }^{3}$ Department of Gastroenterology, La Princesa University Hospital, Madrid, Spain

${ }^{4}$ Department of Anesthesia, Puerta de Hierro University Hospital, Majadahonda, Spain

${ }^{5}$ Center of Endoscopic Research and Therapeutics, University of Chicago Medical Center, Chicago, Illinois, USA

${ }^{6}$ Department of Surgery, Infanta Sofía Hospital, Alcobendas, Spain

\section{References}

1 Forsell P, Hallberg D, Hellers G. Gastric banding for morbid obesity: initial experience with a new adjustable band. Obes Surg 1993; 3: 369-374

2 Stroh C, Hohmann U, Will U et al. Experiences of two centers of bariatric surgery in the treatment of intragastrale band migration after gastric banding-the importance of the German multicenter observational study for quality assurance in obesity surgery 2005 and 2006. Int J Colorectal Dis 2008; 23: $901-908$

3 Weiss H, Nehoda H, Labeck B et al. Gastroscopic band removal after intragastric migration of adjustable gastric band: a new minimal invasive technique. Obes Surg 2000; 10: 167 - 170

4 Neto MP, Ramos AC, Campos JM et al. Endoscopic removal of eroded adjustable gastric band: lessons learned after 5 years and 78 cases. Surg Obes Relat Dis 2010; 6: 423-427

\section{Bibliography}

DOI http://dx.doi.org/

10.1055/s-0031-1291520

Endoscopy 2012; 44: E40-E41

(c) Georg Thieme Verlag KG

Stuttgart · New York

ISSN 0013-726X

\section{Corresponding author}

\section{A. Herreros de Tejada, MD}

Department of Gastroenterology

Hospital Universitario Puerta de Hierro

Majadahonda

Joaquin Rodrigo 2

28220 Majadahonda

Spain

Fax: +34-91-1917807

albertoherreros@yahoo.com 